\title{
Development of Imaging Probes for Fracture Healing Response
}

Center for Bone Biology, Vanderbilt University Medical Center, Nashville, TN 37232

The Masonic Medical Research Institute, Utica, NY 13501

Khanh Ha, ${ }^{*}$ Stephanie N. Moore-Lotridge, ${ }^{*}$ Khada Nagi, Sa Lay Wah, Jonathan G. Schoenecker, and Jason R. McCarthy

\section{1| Introduction}

- More than 16 million fractures are treated in the United States each year

- $10 \%$ of fractures are complicated by delayed union or non-union

- Fractures create five primary problems: bleeding, susceptibility to infection, disproportionate interfragmentary strain, bone hypoxia, and an inability to bear weight

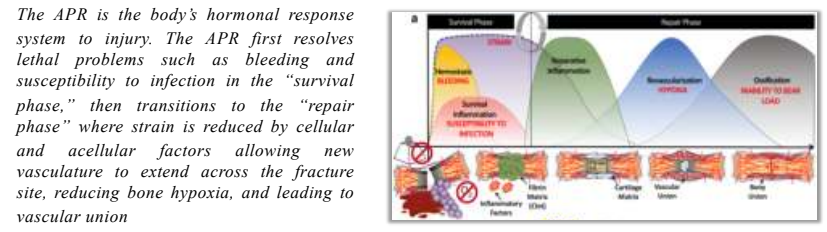

site, reducing bone hypoxia, and leading

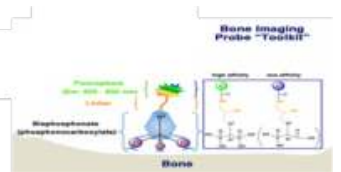

- The goal of this study was to develop and validate new imaging agents that enable high-resolution near-infrared fluorescence (NIRF) imaging of bone fracture acute phase response

\section{2| Background}

- Acute Phase Response: The body's response to fracture injury

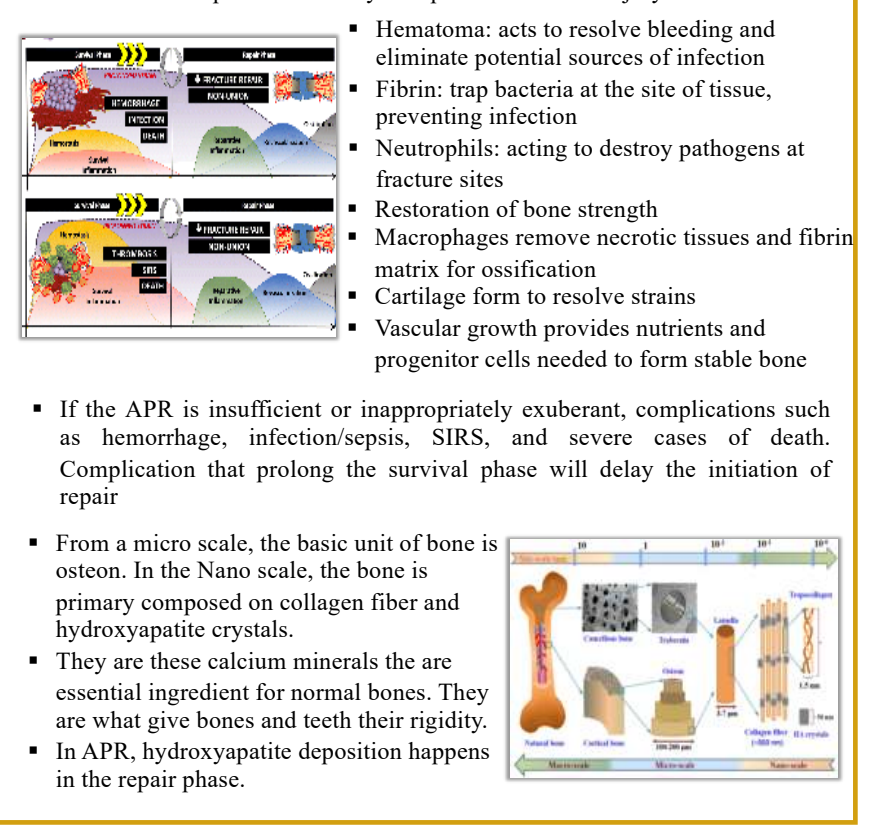

\section{3| Synthesis of CyAl 5.5}

- Endgroup

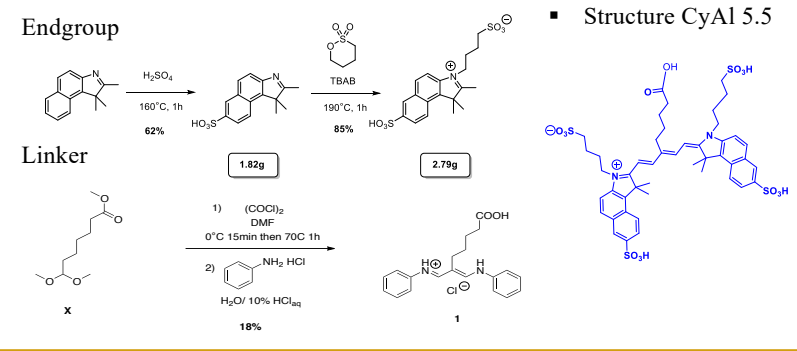

\section{4| Coupling Strategy}

- Carboxylic acid activation with NHS - Coupling of with Pamidronic Acid

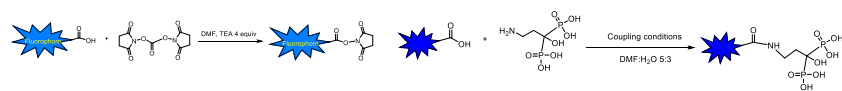

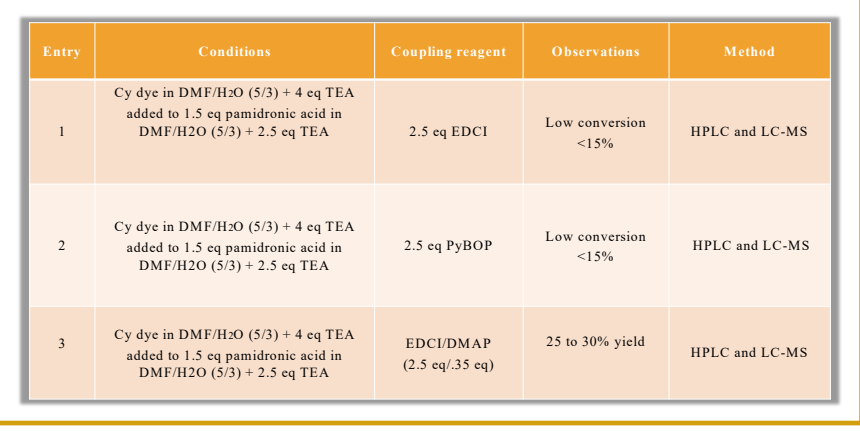

\section{5 | Hydroxyapatite Deposition}

Low doses of BPP-CyAm 7 were injected into mice to image and monitor the healing process of bone in relation to hydroxyapatite exposure (similar results were seen with the BPP-CyAl 5.5 agent)

$$
\text { Week } 1 \text { Week } 2 \text { Week } 3 \text { Week } 4 \text { Week } 5
$$

BPP-CyAm 7

BPP-CyAm 7
with fracture

$B P P-C y A m 7$

without fracture

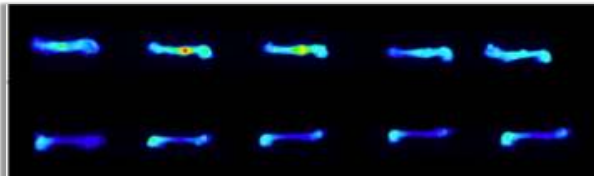

\section{6 | Coupling with Peptides}

- Labeling of binding peptides

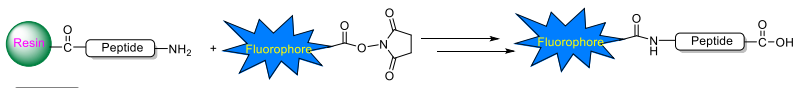
Pepide = collagen or FTP

\section{7 | Matrix Evolution in Transverse Femur}

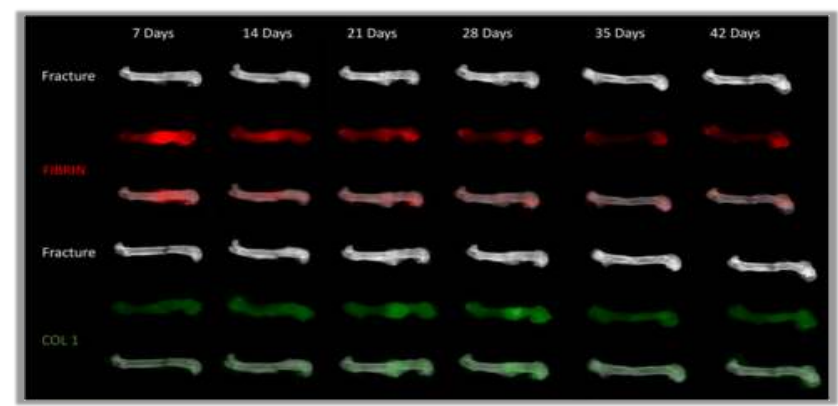

- By 14 days post fracture, the fluorescently labeled fibrin matrix (red) ha begun to be cleared. By 21 days post fracture, the presence of type 1 collagen (green) is pronounced at the site of fracture healing in the form of hard callus. As remodeling of the hard callus occurs through 42 days post fracture, there is a decrease in observable type 1 collagen signal

\section{8 | Conclusion}

- In short, we designed successfully synthesized novel fluorescent probes for molecular imaging of fibrin, collagen and hydroxyapatite

In vivo studies in mice demonstrate the potential for these imaging agents to be applied clinically

Future plan:

- These probes will be used clinically to assess the bone healing proces - We hope to use these dyes in conjugation with other binding agents, allowing us to take a multi-imaging-agent approach to integrate the most significant advancements in fracture biology to create a coherent and unified theory of fracture repair. (i.e. image bone, cartilage, and blood simultaneously)

\section{9 | Acknowledgements}

- McCarthy Group, Jaffer Group, Schoenecker Group

- SUNY Polytechnic Institute, and Masonic Medical Research Institute.

- Funding through National Heart Blood and Lung Institute grants 1R01HL133153. 5R01HL122238, and 1R01HL137913.

(1) 